\title{
A iniciativa da construção do Plano de Dados Abertos do Tribunal de Contas do Rio Grande do Norte através de parceria acadêmica com a Universidade Federal do Rio Grande do Norte
}

\author{
Frederico N. Pranto Filho ${ }^{1}$, Renan Oliveira ${ }^{2}$, Lindemberg Silva Pereira ${ }^{1}$, \\ Joir Ramalho ${ }^{1}$ \\ ${ }^{1}$ Diretoria de Informática \\ Tribunal de Contas do Rio Grande do Norte (TCE-RN) - Natal - RN - Brasil \\ ${ }^{2}$ Instituto Federal de Educação, Ciência e Tecnologia \\ do Rio Grande do Norte (IFRN) - João Câmara - RN - Brasil \\ fredericoprantodtce.rn.gov.br, renan.silva@ifrn.edu.br \\ \{lindemberghsilva, joirramalho\}@tce.rn.gov.br
}

\begin{abstract}
Although there are several advantages and motivations for opening data by public government, there are also some difficulties and barriers to be overcome in order to achieve a Open Government Data (OGD) policy. Studies investigate the main motivations of developers and providers in working with $O G D$, as well as to identify the difficulties faced by them. The general objective of this work is to report the strategy adopted in the preliminary stages of the implementation of the Open Data Plan (ODP) of the Court of Auditors of Rio Grande do Norte (TCE/RN). The construction of the ODP tried to follow, as a working methodology, the directives defined in the ProceDA (Process for Open Data). In addition, we will discuss and share the gains obtained through a partnership between the TCE/RN and the Federal University of Rio Grande do Norte (UFRN).
\end{abstract}

Resumo. Embora haja várias vantagens e motivações para a abertura dos dados pelos órgãos da administração pública, há também algumas dificuldades e barreiras a serem superadas para a efetivação de uma política de Dados Abertos Governamentais (DAG). Estudos investigam as principais motivações dos desenvolvedores e fornecedores em trabalhar com DAG, bem como procuram identificar as dificuldades enfrentada por eles. $O$ objetivo geral deste trabalho compreende relatar a estratégia adotada nos estágios preliminares da implantação do Plano de Dados Abertos (PDA) do Tribunal de Contas do Rio Grande do Norte (TCE/RN). A construção do PDA buscou seguir como metodologia de trabalho as diretivas definidas no ProceDA (Processo para Dados Abertos). Além disso, vamos discutir e compartilhar os ganhos obtidos a partir de uma parceria entre o TCE/RN e a Universidade Federal do Rio Grande do Norte (UFRN).

\section{Introdução}

Uma publicação desenvolvida pelo Tribunal de Contas da União lista cinco motivos para a abertura de dados na Administração Pública [BRASIL 2015] e apresenta algumas razões 
para que as organizações públicas invistam em iniciativas de abertura de dados governamentais. O TCE/RN, desde 2016, disponibilizou um Portal de Dados Abertos ${ }^{1}$ em seu sítio contendo alguns conjuntos de dados no intuito de atender os cinco motivos citados anteriormente que são: (i) transparência na gestão pública; (ii) contribuição da sociedade com serviços inovadores ao cidadão; (iii) aprimoramento na qualidade dos dados governamentais; (iv) viabilização de novos negócios; (v) obrigatoriedade por lei. Dados abertos podem ser considerados como um dos importantes itens para o efetivo exercício do controle social. Controle social é a capacidade da sociedade de intervir nas políticas públicas com o objetivo de garantir direitos [Aguillar 1999].

A iniciativa de Dados Abertos possui diversos pontos positivos, como o potencial de gerar produtos e serviços úteis para a sociedade, fornecer mecanismos de transparência, fortalecer o controle social, a cidadania ativa, a cooperação e melhorias na administração pública além da criação de novas ferramentas, trazendo desenvolvimento e melhoria no bem-estar social [Kucera et al. 2016]. São apontadas ainda como áreas beneficiadas pela iniciativa o empoderamento do cidadão, o desenvolvimento de novos produtos e serviços, inovação, aumento na eficiência e efetividade de serviços governamentais, medição de impacto de políticas, e novos conhecimentos obtidos através da combinação de conjuntos de dados e de padrões em grandes volumes de dados [Open Knowledge International 2017]. Tudo isso é possível quando os dados disponibilizados possuem alto valor agregado [Janssen et al. 2012], possibilitando à própria sociedade explorá-los de maneira mais efetiva do que os próprios donos daqueles dados, que geralmente possuem apenas uma visão limitada sobre eles, utilizando-os para atividades básicas. Ao torná-los públicos, abre-se uma grande gama de possibilidades de utilização e visualização daquelas informações, trazendo benefícios em diversas áreas [Najdenov et al. 2015].

No período da publicação deste artigo, ainda tramita na Câmara dos Deputados o Projeto de Lei 7804/2014 [BRASIL 2014] que institui a Lei de Dados Abertos, estabelecendo o Comitê Gestor de Dados Público junto ao Ministério do Planejamento, responsável pela elaboração do Manual de Dados Abertos da Administração Pública e cria a obrigatoriedade para a disponibilização de dados abertos e de interfaces de aplicações web de forma organizada e estruturada para a União, Estados, o Distrito Federal e Municípios e dá outras providências. Atualmente, relacionado a Dados Abertos, existe o Decreto 8.777/2016 [BRASIL 2016] que institui a Política de Dados Abertos somente para o Poder Executivo Federal. Portanto, os órgãos das esferas estatual e municipal, assim como os Tribunais de Contas dos Estados e Municípios, ainda não são obrigados, por lei, a instituir o PDA ou o Portal de Dados Abertos. Entretanto, alguns portais já são uma realidade em alguns Tribunais de $\operatorname{Contas}^{2,3,4,5}$, Estados $^{6}$ ou Municípios ${ }^{7}$.

Os desafios que envolvem o ecossistema de dados abertos na administração pública já foi discutido em alguns trabalhos [Araújo 2017, Albano and Reinhard 2015, Fontoura 2015, Albano and da Silva Craveiro 2016,

\footnotetext{
${ }^{1}$ Disponível em: <www.apidadosabertos.tce.rn.gov.br $>$

${ }^{2}$ Disponível em: <www.dados.tce.rs.gov.br $>$

${ }^{3}$ Disponível em: <www.tce.es.gov.br/portal-da-transparencia/dados-abertos $>$

${ }^{4}$ Disponível em: <www.portal.tce.pb.gov.br/dados-abertos-do-sagres-tcepb $>$

${ }^{5}$ Disponível em: <www.dadosabertos.tce.mg.gov.br $>$

${ }^{6}$ Disponível em: <www.dados.al.gov.br $>$

${ }^{7}$ Disponível em: <www.dados.natal.br $>$
} 
Albano et al. 2017, Alcantara et al. 2015]. Embora haja várias vantagens e motivações para a abertura dos dados pelos órgãos da administração pública, há também algumas dificuldades e barreiras a serem superadas para a efetivação de uma política de Dados Abertos Governamentais (DAG). Segundo Araújo [2017], há vários fatores que motivam os desenvolvedores e fornecedores a trabalharem com DAG. O trabalho da autora procurou identificar também quais as principais dificuldades enfrentada e os pontos de melhorias no uso de DAG. Os fornecedores são os responsáveis por trabalharem na criação e fornecimento dos DAG. Já os desenvolvedores são usuários que possuem conhecimento técnico capaz de extrair, analisar e desenvolver aplicações que utilizam DAG.

O objetivo deste trabalho é compartilhar os ganhos obtidos a partir da parceria acadêmica entre o TCE/RN e a UFRN na iniciativa da construção do PDA do TCE/RN além de apresentar a estratégia e a metodologia utilizada para a publicação dos dados abertos.

\section{A parceria entre o TCE/RN e o IMD}

O Instituto Metrópole Digital (IMD) é uma Unidade Acadêmica Especializada da UFRN, cujo perfil alinha-se com a missão de fomentar a criação de um Polo Tecnológico em Tecnologia da Informação ${ }^{8}$ no estado do Rio Grande do Norte, abrangendo iniciativas dos setores público, privado e acadêmico.

O IMD atua na formação dos níveis técnico, superior e na pós-graduação. Dentre os cursos de pós-graduação há o Programa de Residência em Tecnologia da Informação aplicado à Área Jurídica e Órgãos de Controle, que inicialmente foi formalizado numa parceria entre o IMD e o Tribunal de Justiça do Rio Grande do Norte e na sequência em outros órgãos. A Residência promove o estudo e o aprofundamento de técnicas e metodologias de Tecnologia da Informação (TI) voltadas ao Poder Judiciário e Órgãos de Controle.

A parceria por meio da Residência entre a UFRN/IMD e o TCE/RN foi publicada no Extrato de Convênio N No 6/2017 [BRASIL 2017]. Os objetivo do convênio são, além da formação e aperfeiçoamento profissional, desenvolver sistemas de inovação tecnológica que irão contribuir na celeridade e qualificação do controle externo exercido pelo TCE/RN, promover avanços em áreas consideradas estratégicas, e estreitar os laços entre o desenvolvimento dos processos internos do órgão e o conhecimento acadêmico.

No total, dez servidores lotados na Diretoria de Informática (DIN) deste tribunal participam como discentes residentes no programa, sendo dois servidores vinculados na ênfase de Infraestrutura e o restante em Desenvolvimento e Business Intelligence (BI). Cada residente deve, como pré-requisito para a conclusão da residência, desenvolver um projeto de TI voltado a atender às necessidades do órgão. Dentre os projetos podemos citar soluções de BI, gestão de projetos, processo de desenvolvimento de software, melhorias em fluxo processuais e auditorias, política de segurança da informação, gerenciamento de acesso de redes sem fio e por fim, transparência. Este último é o tema norteador do nosso projeto.

Alguns projetos da Residência foram priorizados pelo Comitê de Tecnologia da

\footnotetext{
${ }^{8}$ Disponível em: $<$ https://portal.imd.ufrn.br/portal/parque-tecnologico $>$
} 
Informação do TCE/RN e outros puderam ser sugeridos pelos residentes. Este artigo apresenta o projeto de implantação do PDA no TCE/RN que tem como objetivos atualizar, reestruturar e expandir o Portal de Dados Aberto já existente no órgão e principalmente fomentar a cultura de dados abertos a fim de promover um dos pilares do Plano Estratégico dos Tribunais de Contas, o controle social.

Vale ressaltar que, durante o desenvolvimento deste trabalho, outras parcerias se concretizaram. Foram realizadas entrevistas técnicas com instituições que já haviam implantado seu PDA. Uma delas foi a UFRN, através da Superintendência de Informática, setor co-responsável pelo desenvolvimento do PDA na instituição de ensino, que sugeriu a utilização do ProceDA (Processo para Dados Abertos), processo este que lista grande parte das fases e tarefas realizadas por aquela instituição. Assim, após o alinhamento dos objetivos do nosso trabalho com o processo de publicação de dados abertos, decidiuse pela utilização do ProceDA [Silva 2018] como norteador do nosso fluxo de trabalho. Além deste caso, por meio de mais um contato acadêmico na UFRN, fomos apresentados a uma solução de ETL (Extract, Transform, Load) integrada ao CKAN (Comprehensive Knowledge Archive Network) que está sendo desenvolvida por um grupo de pesquisa acadêmica [Macedo et al. 2017, Lopes et al. 2017] .

Percebemos que, mesmo com o projeto ainda em desenvolvimento, há várias oportunidades par que outros órgãos possam consumir os dados custodiados no TCE/RN de forma estruturada. Até o presente momento realizamos contatos com secretarias do governos do estado e setores da sociedade civil organizada. Acreditamos que a partir da promoção desta iniciativa, potencializamos o interesse de futuros consumidores de dados abertos após a futura publicação e divulgação do portal de dados abertos do TCE/RN. Algumas ações de promoção e divulgação do projeto foram realizadas por meio de Workshops, apresentação em eventos e discussões em redes sociais.

Conforme discutido em Araújo [2017], uma forma de garantir a motivação dos fornecedores de dados está ligada ao apoio que as universidades têm oferecido a esses atores. O caráter acadêmico da parceria e o interesse dos fornecedores na participação ativa de grupos de pesquisas envolvidas com a publicação ou o uso dos DAG se mostrou, conforme levantado pela autora, mais uma motivação para o desenvolvimento deste projeto.

\section{O desenvolvimento do Plano de Dados Abertos no TCE/RN como metodologia de trabalho}

Conforme discutido na seção 2, alguns projetos foram definidos e priorizados pelo órgão devido a sua importância estratégica. No entanto, além dos projetos prédefinidos, também foi possível a indicação de novos projetos para serem desenvolvidos na residência. Isto posto, foi criada e apresentada uma proposta para o desenvolvimento do PDA no TCE/RN e após o aceite do projeto pelo Comitê de Tecnologia de Informação do TCE/RN, foi iniciado o planejamento e o desenvolvimento do cronograma inicial de entregas do projeto. Na proposta, foi estabelecido que a metodologia de trabalho seria definida em um processo de publicação de dados, conforme será detalhado nesta seção.

A seguir detalharemos os seguintes pontos: (i) apresentação do processo de publicação de dados abertos; (ii) algumas adaptações do processo no nosso projeto; (iii) o PDA e a estratégia de seleção dos dados; (iv) e a apresentação da solução arquitetural 
desenvolvida.

\subsection{O processo de publicação de dados abertos}

O Manual para Elaboração de Plano de Dados Abertos [BRASIL 2013] editado e publicado em 2013 pelo Ministério do Planejamento, Desenvolvimento e Gestão (MP) destaca as etapas que compõem o processo de construção do PDA. Para as demais tarefas relativas à publicação de dados, optamos por seguir as diretrizes apresentadas na proposta de Silva [2018], intitulada de ProceDA (Processo para Dados Abertos), por se tratar de um processo construído através da observação e compilação das melhores práticas presentes na literatura quanto ao processo de publicação de dados na administração pública. A Figura 1 ilustra suas fases, bem como sua sequência.

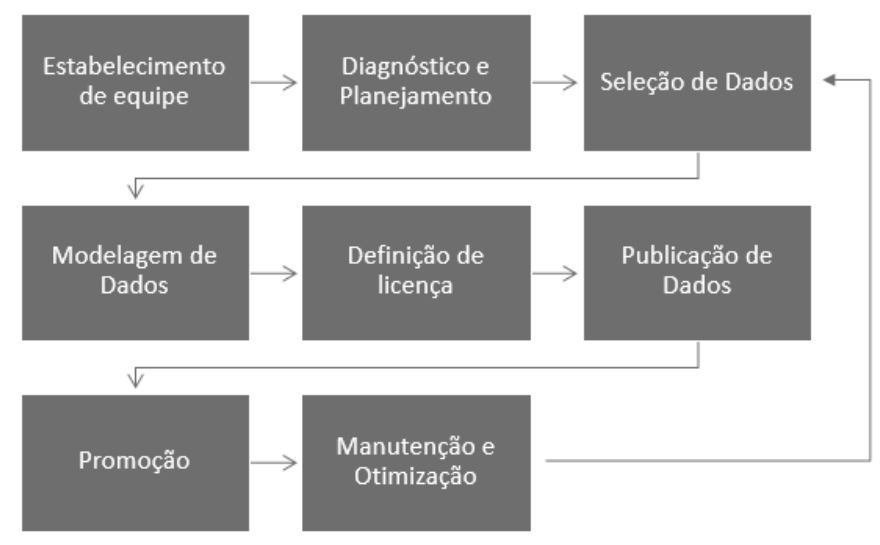

Figure 1. ProceDA e suas fases

O processo possui 8 fases, descritas a seguir:

- Estabelecimento de Equipe: definição da equipe que irá atuar no projeto de Dados Abertos da instituição, com o estabelecimento de papéis.

- Diagnóstico e Planejamento: consiste em avaliar o nível de preparo da instituição, realizar estudos de viabilidade do projeto, estabelecer um Plano de Dados Abertos (PDA), determinar a política de disponibilização de dados, estudo de legislação e outras questões gerais de projeto.

- Seleção de Dados: a instituição deverá selecionar os conjuntos de dados que serão publicados no ciclo atual, observando uma série de questões para o estabelecimento de prioridades, como a confidencialidade dos dados, o valor associado aos conjuntos de dados, benefícios e riscos da disponibilização de determinados dados, entre outros.

- Modelagem de Dados: deverá ser definido o formato de publicação dos dados, que pode ser: Dados Brutos, API ou Dados Conectados. A escolha deve ser baseada nas limitações de infraestrutura e o nível de valor agregado desejado para os dados.

- Definição de Licença: deve-se definir os termos de uso dos dados, que devem ser, resumidamente, de formato aberto, não proprietário, estável e de amplo uso. A publicação de um conjunto de dados sem estabelecer sua licença pode prejudicar sua utilização por parte de terceiros, indo de encontro aos ideais dos Dados Abertos. 
- Publicação de Dados: são estabelecidas tarefas que devem ser realizadas ao se publicar os conjuntos de dados na Web.

- Promoção: são definidas estratégias de promoção dos dados que foram publicados, aumentando a chance de uso pela comunidade.

- Manutenção e Otimização: Deve-se avaliar a necessidade de atualizar conjuntos de dados previamente publicados, além de realizar monitoramento e ajuste das atividades realizadas durante todo o processo, buscando encontrar possíveis pontos de melhoria.

São previstos 5 papéis para a execução das diferentes tarefas associadas ao processo. Eles são descritos a seguir:

- Órgão Central de Suporte e Controle das Ações (OCSCA): órgão que ficará responsável por auxiliar e coordenar o processo de abertura de dados, removendo eventuais obstáculos e problemas que surgirem durante o caminho, bem como articulando todas as unidades que irão abrir seus dados.

- Diretor: Responsável pela iniciativa e principal interessado na abertura de dados. Geralmente é um Executivo da alta gestão, que pode ou não estar diretamente associado ao OCSCA.

- Coordenador: Responde pela execução do processo de abertura e acompanhará de perto as tarefas executadas.

- Gestor Negocial: Tem a capacidade de identificar dados importantes que podem ser abertos, os dados de risco e dados confidenciais.

- Gestor Técnico: Responde pelas ações técnicas do processo de abertura, como extração, elaboração de conjuntos de dados e sua apresentação.

Maiores informações sobre o ProceDA e um detalhamento da lista de tarefas de cada fase do processo, assim como os papéis responsáveis em executá-las, podem ser encontrados em Silva [2018].

\subsection{Adaptações realizadas no processo}

O processo definido no ProceDA sofreu algumas alterações no desenvolvimento das primeiras fases devido, principalmente, à forma com a qual este projeto foi idealizado. Como discutido no início deste artigo, o Decreto 8.777 institui a Política de Dados Abertos do Poder Executivo Federal. Desta forma, por força de decreto, os órgãos em questão tiveram de priorizar seus projetos internos a fim de atender o dispositivo legal. Acreditamos que a fase de Estabelecimento de Equipe, Diagnóstico e Planejamento e a primeira iteração da fase Seleção de Dados, provavelmente, puderam ser bem definidas nesses casos.

No nosso caso, a provocação inicial para o desenvolvimento do projeto não veio, necessariamente, por via legal ou por planejamento institucional. A iniciativa partiu da necessidade do desenvolvimento de um projeto acadêmico vinculado às necessidade do TCE/RN. Identificamos então uma oportunidade de fomentar a cultura dos dados abertos na instituição, além de contribuir para um dos pontos estratégicos definidos no Plano de Gestão (2016 - 2017) [ATRICON 2016] desenvolvido pela ATRICON (Associação os Membros dos Tribunais de Contas do Brasil). Neste documento, um dos objetivos estratégicos apresentados é "Estimular a transparência das informações das decisões e da gestão dos Tribunais de Contas", que apresenta também duas iniciativas: (i) Apoiar 
a instituição da Política Nacional de Fomento ao Controle Social e Dados Abertos pelos Tribunais de Contas; (ii) e apoiar a implantação ou o aprimoramento de ações destinadas a ampliar a transparência da gestão dos Tribunais de Contas. Assim, acreditamos que este projeto está alinhado com os objetivos estratégicos dos Tribunais de Contas.

Assim, algumas atividades das fases iniciais, como: a definição da equipe, estabelecimento de papéis e elaboração do PDA estão ainda sendo definidos de forma paralela às outras fases iterativas (ver Figura 1). Além disso nem todos os papéis foram completamente definidos até a publicação deste artigo.

\subsection{O PDA e a estratégia de seleção dos dados}

De acordo com o Manual para Elaboração de Plano de Dados Abertos [BRASIL 2013], o PDA é o documento que visa orientar as ações de implementação e promoção de abertura de dados, inclusive os geoespacializados, que deverão obedecer aos padrões mínimos de qualidade, de forma a facilitar o entendimento e a reutilização das informações. É ele quem organiza o planejamento referente à implantação e racionalização dos processos de publicação de dados abertos nas organizações públicas.

A implementação da Política de Dados Abertos, conforme estabelecido no Decreto 8.777, é realizada meio da elaboração e execução do PDA no âmbito de cada órgão ou entidade da administração pública federal, com isso, até a presente data, observamos a publicação de vários PDAs em diferentes órgãos. O processo de criação do PDA provoca a discussão do tema sobre transparência, dados abertos e controle social em cada órgão. Espera-se que a experiência do TCE/RN possa contribuir com outras iniciativas de organizações afins, conforme discutido em outros trabalhos [Bertin et al. 2017, Melo et al. 2017, Visoli et al. 2017].

O PDA está em desenvolvimento e será submetido à aprovação do Comitê de Tecnologia da Informação do TCE/RN para novas definições, priorizações e adequações.

A estrutura do PDA segue as recomendações do Manual para Elaboração de Plano de Dados Abertos e possui a seguinte estrutura:

- Apresentação: uma seção que explica e resume, de maneira clara e didática, o conteúdo e propósito do documento, com foco no cidadão.

- Introdução: informa que se trata da instituição do Plano de Dados Abertos do órgão, apresentando o normativo aplicável - incluindo padrões de organização e formatação dos dados a serem publicados - cenário institucional e, a seguir, os objetivos do documento.

- Definição dos Dados a Serem Abertos: nesta seção, enumera e apresenta os critérios para definição de que dados devem ser abertos e apresentar como foi feita a priorização.

- Estratégias para Abertura: detalha o passo-a-passo relacionado ao processo de publicação de dados, bem como premissas e requisitos de qualidade mínimos relacionados.

- Catalogação no Portal Brasileiro de Dados Abertos: aborda as responsabilidades relacionadas processo de publicação/catalogação de dados no Portal Brasileiro de Dados Abertos, incluindo o cadastro dos mantenedores dos metadados no portal. 
- Sustentação: detalha as responsabilidades relacionadas à curadoria da publicação de dados e prever formas para garantir a disponibilidade dos dados abertos, bem como a incorporação do processo de abertura dentro da rotina do órgão.

- Monitoramento e Controle: apresenta o processo de monitoramento e controle das ações previstas no Plano, bem como relatório de prestação de contas.

- Plano de Ação: detalha as atividades e responsabilidades relacionadas ao processo de publicação de dados, bem como prazos e requisitos.

Com relação a definição dos dados a serem abertos, planejamos três principais entregas. A primeira entrega será disponibilizada na publicação do portal e tem como principais conjuntos de dados as informações institucionais divididos em cinco grupos: Contratos e Convênios; Despesa e Orçamento; Pessoas; Institucional; e Dados Auxiliares.

$\mathrm{Na}$ segunda e terceira entregas serão disponibilizados alguns dados enviados pelas Unidades Jurisdicionadas do TCE/RN, esses dados são recebidos e custodiados no tribunal. A segunda entrega tratará dos dados sobre a folha de pagamento e cadastro funcional dos servidores ativos, inativos e pensionistas dos jurisdicionados (SIAI DP) e a última entrega relacionados aos dados fiscais (SIAI Fiscal). Essas informações possuem um grande valor para a transparência, governança e controle social, visto que os todos estes dados estariam consolidados em um único local e em formato aberto.

\subsection{Apresentação da solução arquitetural}

A solução para a publicação dos dados abertos no Portal de Dados Abertos do TCE/RN que está sendo desenvolvido neste projeto está baseada no uso do CKAN ${ }^{9}$. O CKAN é uma ferramenta de catálogo de dados abertos, de código aberto, e desenvolvido pela Open Knowledge Foundation ${ }^{10}$, uma organização sem fins lucrativos que promove conhecimento livre. O CKAN é usado por governos nacionais e locais, instituições de pesquisa e outras organizações que coletam muitos dados. No Brasil, destaca-se o Portal Brasileiro de Dados Abertos ${ }^{11}$, um portal disponibilizado pelo governo para que todos possam encontrar e utilizar os dados abertos e as informações públicas.

Para realizar a carga periódica dos dados no portal utilizamos a ferramenta ETL Pentaho Data Integration ${ }^{12}$. A criação da infraestrutura do Pentaho foi realizada por outro projeto de BI da Residencia de TI. Utilizamos a arquitetura já montada nos servidores do tribunal para criar e executar as transformações ETL dos conjuntos de dados. A carga é realizada diretamente para o CKAN através do plug-in CKAN Resource Updater for Pentaho Data Integration (Kettle $)^{13}$.

\section{Conclusão}

O presente artigo apresentou a iniciativa, ainda em andamento, da construção do Plano de Dados Abertos do Tribunal de Contas do Rio Grande do Norte. Este projeto surgiu como pré-requisito de um curso de pós-graduação de nível lato sensu organizado a partir de um termo de cooperação entre o TCE/RN e a UFRN. O ProceDA (Processo para Dados

\footnotetext{
${ }^{9}$ Disponível em: $<$ www.ckan.org $>$

${ }^{10}$ Disponível em: $<$ www.okfn.org $>$

${ }^{11}$ Disponível em: $<$ www.dados.gov.br $>$

${ }^{12}$ Disponível em: <www.pentaho.com/product/data-integration $>$

${ }^{13}$ Disponível em: < github.com/localidata/CKAN-Resource-Updater-for-Pentaho-Data-Integration $>$
} 
Abertos) está sendo utilizado como metodologia de construção do PDA. Além do PDA, o Portal de Dados Abertos está sendo reestruturado e atualizado por meio da plataforma de publicação de dados CKAN. Vale também ressaltar que as parcerias realizadas até o momento, seja com a academia ou com outros órgãos são fundamentais na aplicação dos conhecimentos acadêmicos nos processos da administração pública e mostram que esta relação é favorável para o desenvolvimento de soluções inovadoras.

Contudo, este artigo levanta somente alguns dados e processos iniciais da iniciativa da construção do PDA do TCE/RN, deixando em aberto os possíveis desafios na seleção e modelagem dos dados, as implicações sobre sigilo e privacidade de dados, validação da publicação dos dados no ambiente de produção, avanços e impacto na política de dados abertos governamentais do TCE/RN e estratégias de promoção dos dados abertos para a sociedade por meio de eventos e hackathons.

Como limitação do trabalho destacamos que embora outros tribunais já tenham publicado seus Portais de Dados Abertos não encontramos na literatura experiências sobre a publicação do PDA em outros Tribunais de Contas. Embora há estudos relacionado o nível de transparência dos Tribunais de Contas [da Silva et al. 2016, da Silva et al. 2014], ainda há a necessidade de uma investigação mais detalhada neste quesito.

Por fim, pretende-se com esta iniciativa, incentivar o desenvolvimento de um ecossistema de dados abertos no Estado do Rio Grande do Norte e nos Tribunais de Contas. Contribuindo assim para que essas ações fomentem a transparência e o controle social.

\section{References}

Aguillar, F. H. F. (1999). Controle social de serviços públicos. Max Limonad.

Albano, C. S., Araujo, M. H., and Reinhard, N. (2017). Fatores motivadores e facilitadores dos relacionamentos em redes: como os gestores públicos reconhecem esses fatores em dados governamentais abertos. Navus-Revista de Gestão e Tecnologia, 7(1):73-92.

Albano, C. S. and da Silva Craveiro, G. (2016). Lições aprendidas com a utilização de dados orçamentários em formato aberto: Um estudo exploratório no ecossistema brasileiro. Revista de Gestão e Projetos-GeP, 6(3):17-27.

Albano, C. S. and Reinhard, N. (2015). Desafios para governos e sociedade no ecossistema brasileiro de dados governamentais abertos (dga). Cadernos Gestão Pública e Cidadania, 20(67).

Alcantara, W., Bandeira, J., Barbosa, A., Lima, A., Ávila, T., Bittencourt, I., and Isotani, S. (2015). Desafios no uso de dados abertos conectados na educaçao brasileira. In Anais do Desafio Workshop de Desafios da Computação Aplicada à Educação. CSBC.

Araújo, N. M. (2017). Dados abertos do governo brasileiro: entendendo as perspectivas de fornecedores de dados e desenvolvedores de aplicações ao cidadão. Master's thesis, UFRN.

ATRICON (2016). Plano de Gestão (2016 - 2017). Brasília.

Bertin, P. R. B., Machado, C. D., Visoli, M. C., Drucker, D. P., Pinto, D. M., et al. (2017). A construção do plano de dados abertos de uma organização pública de pesquisa e desenvolvimento e o desafio de uma ciência agropecuária aberta. 
BRASIL (2013). Manual para elaboração de plano de dados abertos.

BRASIL (2014). Projeto de Lei $n^{\circ} 7804 / 2014$. Brasília.

BRASIL (2015). Cinco motivos para a abertura de dados na administração pública.

BRASIL (2016). Decreto $n^{o} 8777 / 2016$. Brasília.

BRASIL (2017). Extrato de Convênio $n^{o}$ 6/2017. Brasília. Seção 3. Diário Oficial da União $\mathrm{n}^{\circ}$ 204, 24 de out. 2017.

da Silva, C. F., dos Santos, E. M. F., Chaves, M. C., Vaz, W., and Balaniuk, R. (2014). Dados abertos: uma estratégia para o aumento da transparência e modernização da gestão pública. Revista do TCU, (131):22-29.

da Silva, W. G., Maciel, C., de Castilho, F. B. M., and Girata, N. N. H. (2016). Um método quantitativo para avaliar a adoção de dados abertos nos tribunais de contas do brasil. iSys-Revista Brasileira de Sistemas de Informação, 9(1):33-57.

Fontoura, M. C. (2015). Hackeando dados abertos no brasil: motivações e práticas. Cambiassu: Estudos em Comunicação, 15(17).

Janssen, M., Charalabidis, Y., and Zuiderwijk, A. (2012). Benefits, adoption barriers and myths of open data and open government. Information Systems Management (ISM), 29(4):258-268.

Kucera, J., Chlapek, D., Klímek, J., and Necasky, M. (2016). Methodologies and best practices for open data publication. In Proceedings of the Dateso 215, pages 52-64. CEUR-WS.

Lopes, F., Barros, A., and Almeida, A. (2017). Processo automatizado de etl para dados abertos: Um estudo de caso do suap e ckan.

Macedo, J., Cacho, N., and Lopes, F. (2017). A comparative study of tools for smart cities open data publication and management. In Proceedings of the 2017 IEEE Summer School on Smart Cities (IEEE S3C 2017).

Melo, J. d. S., Melis, M. F. M. d. S., et al. (2017). Análise da implementação da política de dados abertos no âmbito do poder executivo federal.

Najdenov, B., Pejchinoski, H., Cieva, K., Jovanovik, M., and Trajanov, D. (2015). Open financial data from the macedonian stock exchange. In Proceedings of the 6th Information and Communication Technologies Innovations 2014 conference, pages 115-124. Springer Verlag.

Open Knowledge International (2017). Open data handbook. Disponível em: ¡http: / / opendatahandbook . orgi. Acesso em 13 Out., 2017.

Silva, R. O. (2018). Uma proposta de processo para implantação de dados abertos em instituições públicas brasileiras. Master's thesis, UFRN.

Visoli, M. C., Bertin, P. R. B., Silva, A. R., Machado, C. R. D. L., Gonzales, L. E., Vacari, I., et al. (2017). Acesso aberto na embrapa: breve histórico, avanços recentes e desafios. 